\title{
Fungos associados ao processo de decomposição foliar: 2 anos de estudo
}

\author{
Anelise Kappes MARQUES ${ }^{[1 ; *]}$; Jessica Barros Aguiar SILVA ${ }^{[1]}$; Amaraina Maia ARMIATO ${ }^{[1]}$; Laciene Marinho \\ SANTOS $^{[1]}$ e Paula Benevides MORAIS ${ }^{[2]}$ \\ ${ }^{[1]}$ Universidade Federal do Tocantins, Campus Palmas. Laboratório de Microbiologia Ambiental e Biotecnologia. Avenida NS 15, 109 Norte - \\ Plano Diretor Norte, 77001-090. Palmas-TO, Brasil. Email: jessicabbarros@hotmail.com; amaraina.maia@gmail.com; \\ lacienemarinho@hotmail.com \\ ${ }^{[2]}$ Professora do programa de Pós-Graduação Doutorado em Biodiversidade e Biotecnologia da Amazônia Legal (BIONORTE). Laboratório de \\ Microbiologia Ambiental e Biotecnologia. Avenida NS 15, 109 Norte - Plano Diretor Norte, 77001-090. Palmas-TO, Brasil. E-mail: \\ moraispb@uft.edu.br
}

\begin{tabular}{|c|c|}
\hline & RESUMO \\
\hline & \multirow{12}{*}{$\begin{array}{l}\text { O processo de decomposição da matéria orgânica em córregos de baixa ordem, } \\
\text { realizado em grande parte pelos fungos, é importante para o fornecimento de energia } \\
\text { a diversos níveis tróficos neste ecossistema. O presente trabalho avaliou a variação } \\
\text { temporal de bolores e leveduras associados ao processo de decomposição foliar em } \\
\text { um córrego de baixa ordem no cerrado tocantinense. Detrito vegetal misto foi } \\
\text { coletado do aporte vertical da vegetação ripária e colocado em sacos de malha grossa } \\
\text { e submersos por } 30 \text { dias no córrego Buritizal. O isolamento e contagem dos fungos } \\
\text { filamentosos e leveduras foi realizado a partir do macerado de discos foliares em } \\
\text { caldo peptonado e semeado em placas de petri contendo meio BDA e YMA } \\
\text { incubados por } 30 \text { dias. As contagens de leveduras variaram de } 1,42 \text { logUFC.gMOF } \\
\text { em junho de } 2011 \text { a } 5,90 \text { logUFC.gMOF em abril de } 2012 \text {. Os fungos filamentosos } \\
\text { variaram de } 1,98 \text { logUFC.gMOF em julho de } 2011 \text { a } 6,27 \text { logUFC.gMOF em março } \\
\text { de } 2013 \text {. As maiores contagens de fungos filamentosos e leveduras ocorreram em } \\
\text { ambos períodos chuvosos porém somente os filamentosos apresentam diferença } \\
\text { estatisticamente significativa e podem estar associadas as enxurradas, quando há o } \\
\text { escoamento da serrapilheira e solo das margens com consequente maior entrada de } \\
\text { geofungos e demais sapróbios associados ao aporte horizontal. }\end{array}$} \\
\hline $0 / 11 / 2015$ & \\
\hline$/ 12 / 2015$ & \\
\hline & \\
\hline & \\
\hline & \\
\hline decom & \\
\hline microbiota & \\
\hline fungos filamentosos & \\
\hline leveduras & \\
\hline *Autor para correspondência & \\
\hline & \\
\hline
\end{tabular}

\section{Fungi associated with the organic matter decomposition process- a two-year work}

\begin{abstract}
The organic matter decomposition process in first order streams is realized mostly by fungi and it is important to provide energy to various trophic levels in this ecosystem. The present work assesses the temporal variation of molds and yeasts, which are collaborators in the leaf decomposition process, in first order stream of Cerrado vegetation in Tocantins State. Plant litter was collected from vertical input of riparian vegetation and inserted in litterbags. Subsequently, they were submerged for 30 days in the Buritizal stream. The isolation and counting of filamentous fungi and yeasts was done from the maceration of leaf discs in peptone solution and streaking in petri disks containing BDA and YMA media for 30 days. The yeasts counting vary from 1,42 $\log$ UFC.gMOF in June, 2011 to 5,90 $\log$ UFC.gMOF in April, 2012. The filamentous fungi vary from 1,98 $\log$ UFC.gMOF in June, 2011 to 6,27 $\log$ UFC.gMOF in March, 2013. The highest quantities of fungi and yeasts were observed in rainy periods and they can be due to floods when the drain of the litter or the soil margin happened. As a result, there is a major entrance of terrestrial and saprobic fungi associated in the horizontal input.
\end{abstract}

Index terms: leaf decomposition; fungus microbiota; filamentous fungi; yeasts

Copyright: () 2015 JBFS all rights. This is an open-access article distributed under the terms of the Creative Commons Attribution License, which permits unrestricted use, distribution, and reproduction in any medium, provided the original author and source are credited.

Financiamento: Os autores reportam que houve suporte e auxílio financeiro pela Coordenação de Aperfeiçoamento de Pessoal de Nível Superior (CAPES), AUXPE-PNPD2682/2011/processo 23038.007227/2011-15 e Conselho Nacional de Desenvolvimento Científico e Tecnológico (CNPq), processo 550912/2010-0.

Conflito de interesse: Os autores declaram que não há conflito de interesse.

Como referir esse documento (ABNT):

MARQUES, A. K.; SILVA, J. B. A.; ARMIATO, A. M.; SANTOS, L. M.; MORAIS, P. B. Fungos associados ao processo de decomposição foliar: 2 anos de estudo. Journal of Bioenergy and Food Science, Macapá, v.2, n.4, p.145-151, out./dez., 2015. http://dx.doi.org/10.18067/jbfs.v2i4.58 


\section{INTRODUÇÃO}

A decomposição do detrito foliar é um processo ecológico importante em riachos, principalmente naqueles onde a sombra formada pela vegetação ripária limita a produção primária pela fotossíntese, tornando assim os materiais vegetais alóctones (folhas, frutos, galhos e troncos) a principal fonte energética destes ecossistemas [1]. $\mathrm{O}$ processo de decomposição da matéria orgânica em córregos de baixa ordem é relevante para o fornecimento de energia a diversos níveis tróficos e a capacidade de utilização desta energia pelos organismos aquáticos depende de fatores ambientais tais como disponibilidade de nutrientes nas águas e no próprio detrito foliar e da temperatura [2].

A decomposição em ecossistemas aquáticos é um processo quase que essencialmente microbiológico envolvendo especialmente fungos e bactérias e é dividido em três fases distintas: a lixiviação, sendo uma rápida perda de peso devido à lavagem de componentes solúveis das folhas mortas; o condicionamento, isto é, a modificação da matriz de folha por micro-organismos como resultado das atividades enzimáticas e a fragmentação, ou seja, o desmembramento físico da matéria orgânica particulada grossa, mediada pela alimentação do invertebrado triturador [3]. No processo de condicionamento microbiano destacamse os fungos, que inicialmente colonizam a matéria orgânica iniciando a decomposição foliar através de um ataque aos polissacarídeos estruturais com a produção de enzimas extracelulares, com papel relevante no aumento da palatabilidade foliar para os macroinvertebrados fragmentadores [4].

Os fungos são relevantes decompositores de matéria orgânica submersa, mostrando-se, nos primeiros estágios de decomposição, muitas vezes mais ativos que as bactérias. Os fungos possuem formas leveduriformes ou filamentosas. As leveduras aquáticas são fungos formados por uma única célula eucarionte, têm forma esférica, oval ou cilíndrica e constituem um grupo polifilético, produtoras de enzimas capazes de degradar diversos tipos de substratos. Em sua maioria são classificadas como ascomicetos, predominantes de ambientes aquáticos e com matéria orgânica em decomposição [5]. Já os fungos filamentosos são pluricelulares dotados de tubos germinativos que se alongam na fração apical formando longos filamentos denominados hifas que em conjunto formam o micélio [6].

No Brasil, trabalhos sobre fungos aquáticos em ambientes lóticos ainda são escassos. Considerando-se a importância da microbiota fúngica para o processo de decomposição de detritos vegetais em ecossistemas aquáticos, o presente trabalho avaliou a variação temporal de bolores e leveduras associados ao processo de decomposição foliar em um córrego de baixa ordem no cerrado tocantinense.

\section{MATERIAL E MÉTODOS}

O trabalho foi realizado no córrego Buritizal, localizado na Sub-bacia do ribeirão Taquaruçu Grande localizado na parte centro sul do município de Palmas, estado do Tocantins entre os paralelos $10^{\circ} 10^{\prime} 10^{\prime \prime}$ e $10^{\circ} 25^{\prime} 18^{\prime \prime}$ de Latitude Sul e os meridianos $48^{\circ} 03^{\prime}$ 57' e $48^{\circ} 23^{\prime}$ '03' de Longitude Oeste de Greenwich. O leito desse riacho é composto por areia, seixos grossos em proporções entre 30:70 a 50:50, com acúmulo de detritos vegetais (p. ex. folhas, troncos, galhos e frutos), principalmente nas áreas de remanso. Alternam-se áreas de remanso largas e profundas (entre 1,0 a 1,20 m) com áreas extensas ( 15 a $30 \mathrm{~m})$, estreitas $(0,30$ a $0,70 \mathrm{~m})$ e menos profundas $(0,30$ a $0,80 \mathrm{~m})$. Raízes de plantas fixadas às margens e macrófitas aquáticas nas áreas de rápidos também compõem os substratos disponíveis. Esta área ocupa um total de 46.307,31 ha, o que representa $19,1 \%$ do município de Palmas sendo que $73,67 \%$ estão inseridos na Área Proteção Ambiental - APA Serra do Lajeado. A microbacia do córrego Buritizal compõe cerca de 1,85\% da SubBacia do Taquaruçu Grande, e os riachos componentes são de primeira e segunda ordem. $\mathrm{O}$ comprimento do canal principal é de 1,37 km com uma rede de drenagem de $1,43 \mathrm{~km}$ e sinuosidade média de 1,87 [7] (Figura 1).

A sub-bacia do Taquaruçú Grande apresenta águas ligeiramente mornas, ácidas e bem oxigenadas com temperatura média de $28^{\circ} \mathrm{C}, \mathrm{pH}$ médio de 6,97 e oxigenação variando entre 4,5 à 10,8 mg. $\mathrm{L}^{-1}$, além disso possui padrões comportamentais diferentes de sólidos totais dissolvidos, condutividade elétrica, nitrogênio total, nitrato, nitrito, amônia e fósforo entre as estações secas e chuvosas [8].

$\mathrm{O}$ processo de decomposição foi acompanhado durante dois anos (dezembro de 2011 a março de 2013), utilizando detritos foliares pertencentes à flora da mata ripária dos córregos selecionados. Para coleta deste detrito foi utilizada metodologia proposta por Gonçalves e Callisto [9] onde coletores interceptaram (durante um mês) folhas senescentes provenientes do aporte vertical. Este material foliar foi seco ao ar e porções de aproximadamente 2,5 gramas, foram incubadas em sacos de decomposição $(10 \mathrm{~mm}$ de malha e dimensões de $10 \times 15 \mathrm{~cm}$ ) durante 30 dias. Este procedimento foi repetido a cada 30 dias. O experimento foi instalado em cinco pontos amostrais ao longo do córrego, com uma distância 
de 10 a 20 metros entre os pontos. Em cada ponto foram instalados três sacos de decomposição em

cada ponto totalizando 15 amostras por período.

Figura 1. Sub-bacia do Taquarucu Grande, localização do ponto Amostral no Córrego Buritizal.

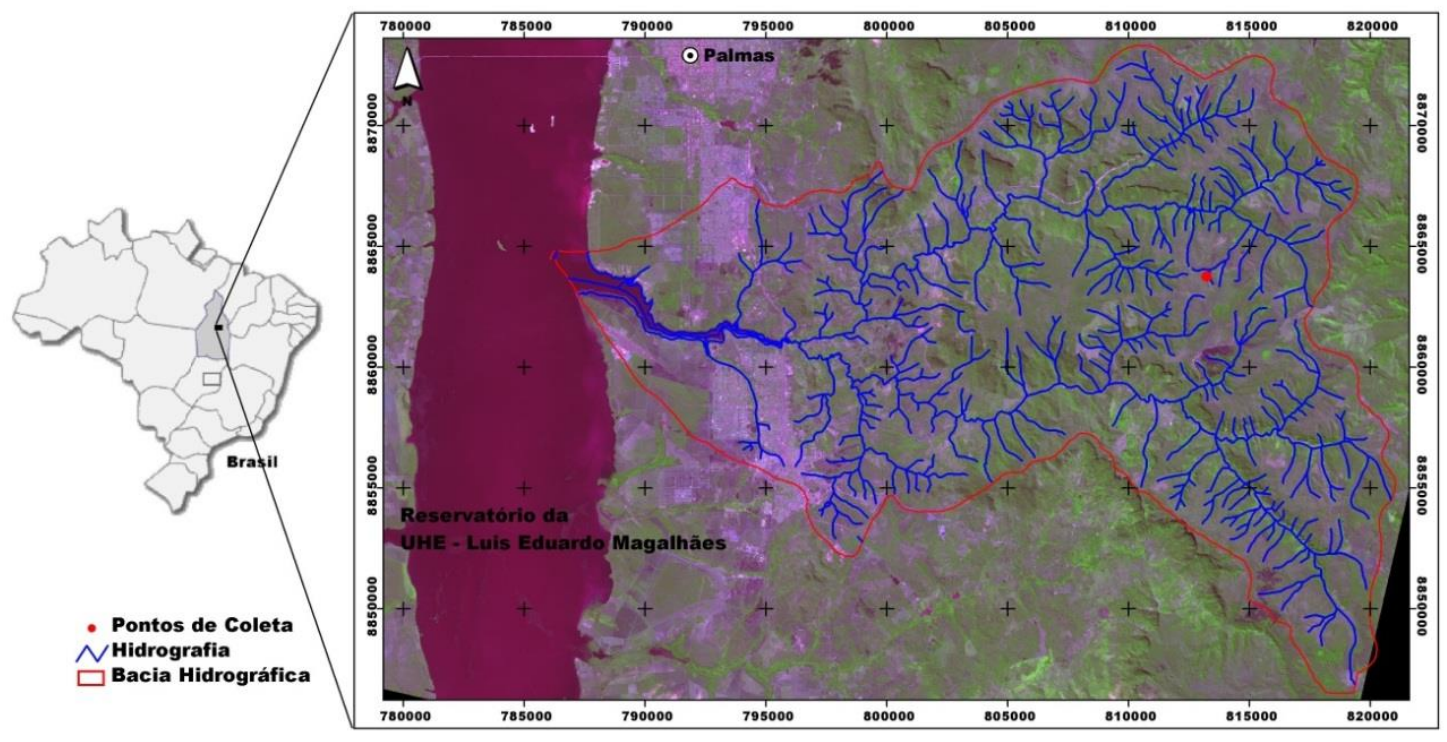

Fonte:Elaborado pelo autor

Após 30 dias os sacos foram retirados do córrego colocados em sacos plásticos e transportados para o laboratório em caixas térmicas com gelo. No laboratório, as folhas foram removidas dos sacos e lavadas individualmente com água destilada. Destas folhas foram retirados 2 conjuntos de discos (5 unidades de discos com $2 \mathrm{~cm}$ de diâmetro) sendo que um conjunto foi utilizado para análise de peso seco livre de cinzas (AFDM) para quantificação da matéria orgânica foliar [10], e o outro conjunto para realizar as contagens fúngicas.

Já para a contagem fúngica o jogo de disco retirado das folhas foi macerado em $9 \mathrm{~mL}$ água peptonada (1:10) e retiradas alíquotas de $100 \mu \mathrm{l}$ da suspensão que para inoculação, em três diluições $\left(10^{-1}, 10^{-2}, 10^{-3}\right)$. As alíquotas de água peptonada foram semeadas pour plate em placa de petri contendo meio BDA com rosa de bengala (peptona $0,5 \%$, glicose $1,0 \%, \mathrm{~K}_{2} \mathrm{HPO}_{4} 0,1 \%, \mathrm{MgSO}_{4} .7 \mathrm{H}_{2} \mathrm{O}$ $0,05 \%$, Rosa de bengala $0,033 \%$, Ágar $1,5 \%$ ) para crescimento dos fungos e para crescimento das colônias de leveduras uma alíquota de cada diluição da água peptonada foi semeada em meio YMA (peptona 0,5\%, extrato de levedura 0,3\%, 27 glicose $1,0 \%$, extrato de malte $0,3 \%$, ágar $2,0 \%$ ), contendo $200 \mathrm{mg} / \mathrm{L}$ de cloranfenicol em placa de petri.

Assim para a contagem de geofungos as placas foram mantidas em estufa incubadora por até 5 dias a $25^{\circ} \mathrm{C}$, sendo posteriormente contadas as colônias existentes e as placas foram identificadas com códigos de procedência dos discos das folhas, as colônias de geofungos foram contabilizadas e devidamente registradas em caderno de acordo com seu crescimento em cada diluição. Já as placas para contagens de leveduras foram mantidas em estufa incubadora a $25^{\circ} \mathrm{C}$ e realizada contagem em 3,5 e 7 dias, a contagem de leveduras foi realizada a partir da descrição e tipificação das colônias em cada diluição. Assim, as colônias que apresentaram crescimento em placas, foram descritas de por suas características de tamanho, elevação, bordas, cor e aspecto [11]. A cada tipo colonial diferente foi atribuído um código de identificação.

Os dados de precipitação foram obtidos através da Estação Meteorológica Palmas INPE/UFT/CUP.

Os dados foram tratados com Análise de Variância (ANOVA) e Teste de Tukey para testar as diferenças de contagem populacionais microbianas no período de seca e chuva. Foi utilizado o software ASSISTAT [12].

\section{RESULTADOS E DISCUSSÃO}

Devido às características climáticas, o córrego Buritizal apresentou durante o estudo, período de estiagem e chuva bem distintos, ocorrendo de outubro a abril e maio a setembro respectivamente. Como os fatores abióticos tendem a serem influenciados pela precipitação tem-se que as chuvas, no período amostral analisado, ocorreram com maior pluviosidade de dezembro de 2011 a abril de 2012 retornando em outubro de 2012 a março de 2013. A máxima pluviosidade ocorreu em 
dezembro de 2012 atingindo $397.764 \mathrm{~mm}$; nos não houve precipitação (Figura 2). meses de julho, agosto e setembro de 2011 e 2012

Figura 2: Pluviosidade (mm) observada no período do estudo

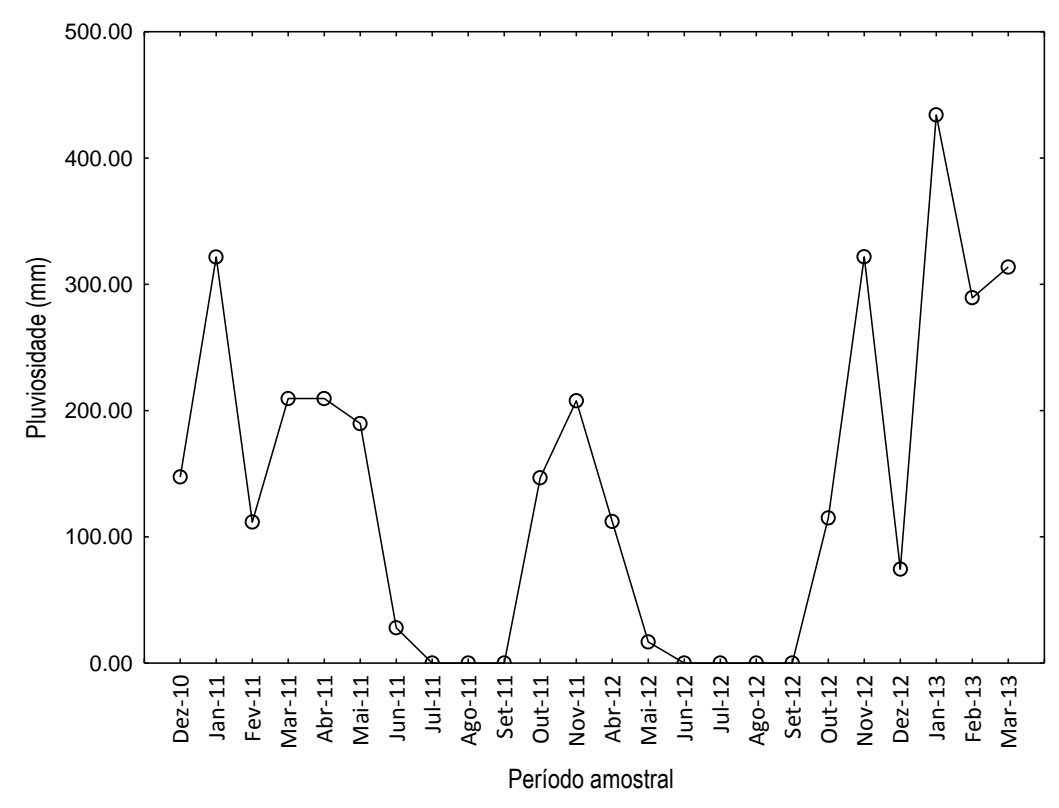

O crescimento de colônias de leveduras associados ao processo de decomposição foliar foi observado durante todo o período de estudo. A densidade de leveduras variou ao longo do tempo de estudo. De acordo com os dados coletados, os maiores picos na densidade de leveduras foram observados nos meses de abril e junho de 2012 e nos meses de janeiro, fevereiro e março de 2013. Nesses meses as contagens foram acima de 5
logUFC.gMOF, sendo em abril de 2012 observada a maior contagem de todo o período de estudo chegando a 5,9 logUFC.gMOF. Em nosso estudo os picos de densidade de leveduras ocorreram tanto período chuvoso quanto nos meses de seca. Os dados coletados e registrados no período de dois anos no córrego Buritizal estão demonstrados na Figura 3.

Figura 3. Contagem de leveduras (LogUFC.gMOF) no período amostral de dezembro de 2010 a janeiro de 2013

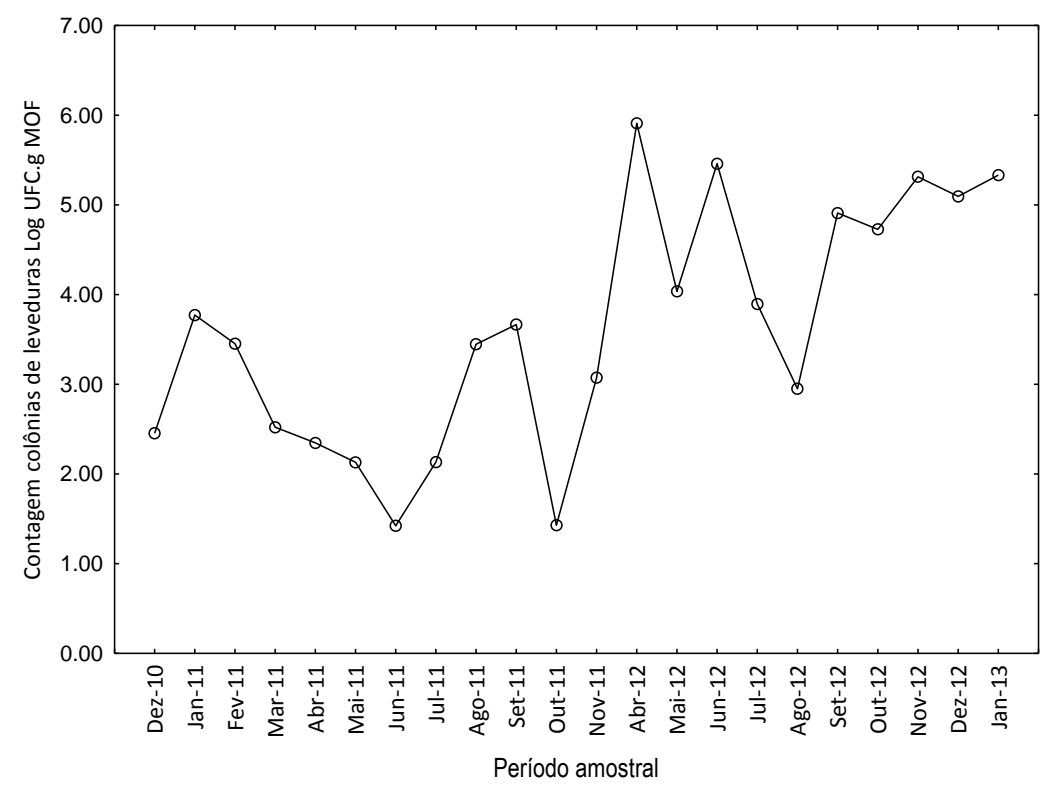

No período compreendido entre dezembro de 2010 a novembro de 2011 as densidades de leveduras foram menores que no período de abril de 2012 a março de 2013, demonstrando que houve um 
aumento no número de colônias no nesse período. Esse aumento da densidade de leveduras pode ter relação com pluviosidade. Dados de pluviosidade do Laboratório de Meteorologia da Universidade Federal do Tocantins (Figura 2) registraram no primeiro trimestre de 2013 um volume de chuva foi maior que nos anos anteriores de 2012 e 2011. Nosso estudo demonstrou que, apesar das populações de leveduras estarem presentes tanto no período chuvoso quanto no período seco, densidades de leveduras maiores ocorreram em períodos de maiores volumes de chuva.

Estudos realizados por Silva-Bedoya et al. [13] sobre diversidade de leveduras associado a água, demonstrou altas contagens de colônias de leveduras nas amostras coletadas em um lago. A presença de micro-organismos em ambientes aquáticos, também foi também observada por Wantzen et al [14], em seu estudo sobre decomposição da matéria orgânica em córregos tropicais, sendo registrado um acúmulo de microorganismos em superfície de material foliar submerso.

Os fungos filamentosos variaram de 1,98 logUFC.gMOF em julho de 2011 a 6,27 logUFC.gMOF em março de 2013. Suas maiores contagens ocorreram nos meses de março/13 e dezembro/12 (6,278 logUFC.gMOF e 6,128 logUFC.gMOF, respectivamente) e os meses com menores índices em julho e agosto/11 (1,98 logUFC.gMOF e 2,44 logUFC.gMOF, respectivamente). Houve presença de fungos filamentosos associados ao processo de decomposição foliar durante todos os meses de estudo, sendo que as contagens estão diretamente relacionadas com os índices pluviométricos. As contagens de fungos filamentosos apresentaram um aumento considerável no segundo ano (Figura 4).

Figura 4. Contagem de fungos filamentosos (LogUFC.gMOF) no período amostral de dezembro de 2010 a janeiro de 2013

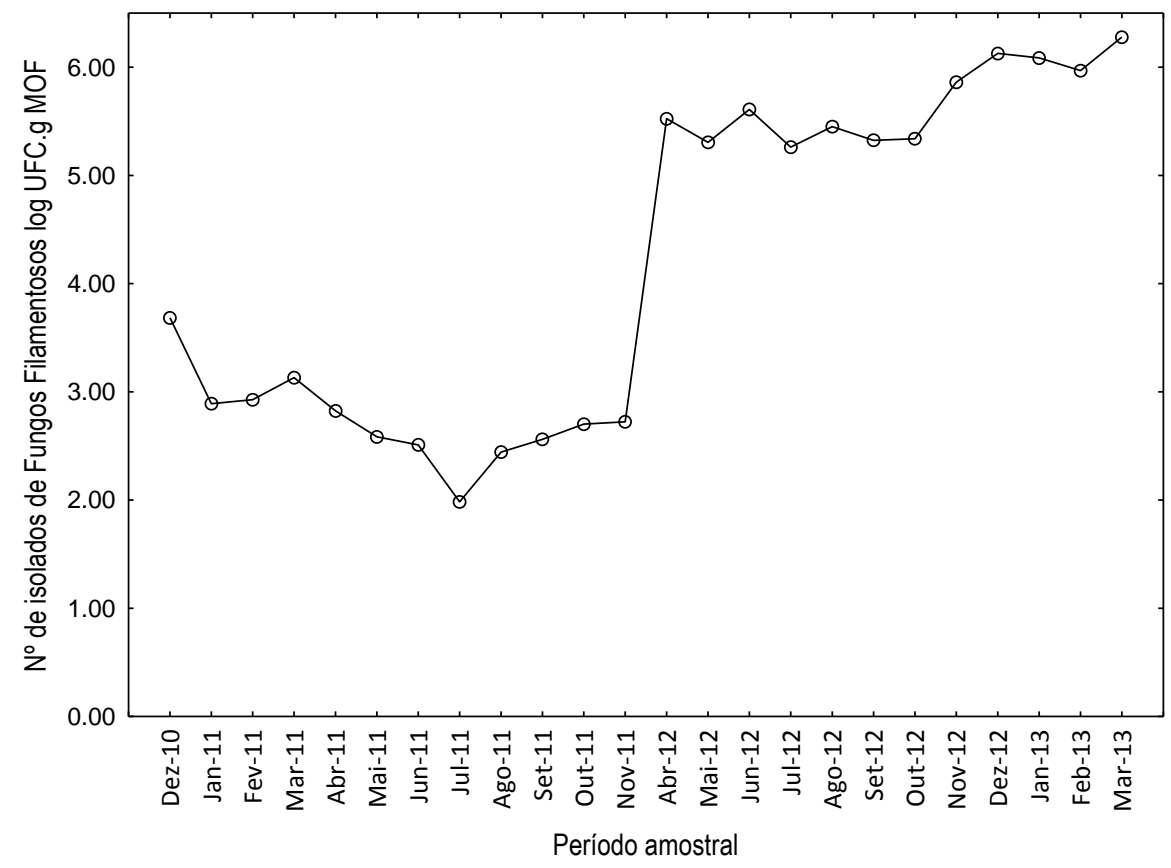

Os maiores valores de contagens de fungos apresentados no período chuvoso podem estar associados às enxurradas provocadas pela chuva, época em que há o escoamento da serrapilheira e do solo das margens do córrego e, consequentemente, a entrada de geofungos (fungos de origem terrestre) e demais sapróbios associados ao detrito vegetal pertencente ao aporte horizontal. No trabalho de Schoenlein-Crusius et al. [15] foi observada grande similaridade entre a micobiota identificada no processo de decomposição em meio aquáticos e fungos originalmente terrestres.
Embora os geofungos sejam considerados terrestres eles possuem alta capacidade de adaptação à vida aquática, com algumas espécies sendo consideradas aquáticas facultativas, pois podem esporular nesse meio $[16,17]$.

As contagens das leveduras não apresentaram diferença estatisticamente significativa entre os períodos de seca e chuva (ANOVA, F=1,73, $\mathrm{p}=0,2199)$. Já a os fungos filamentosos apresentaram nível de significância a $1 \%$ para os tratamentos (ANOVA, $\mathrm{F}=14,4474, \quad \mathrm{p}=0,0029$ ), diferenciando, portanto, os períodos de seca e chuva conforme verificado na Tabela 1. 
Tabela 1. Médias e Coeficiente de Variação dos tratamentos para período seco e chuvoso

\begin{tabular}{lccc}
\hline $\begin{array}{l}\text { Médias de } \\
\text { tratamentos }\end{array}$ & $\begin{array}{c}\text { Tratamento } \\
\text { 1: chuva }\end{array}$ & $\begin{array}{c}\text { Tratamento } \\
\text { 2: seca }\end{array}$ & CV \\
\hline $\begin{array}{l}\text { Fungos } \\
\text { filamentosos }\end{array}$ & $0,49300 \mathrm{a}$ & $0,53523 \mathrm{~b}$ & $5,29 \%$ \\
$\begin{array}{l}\text { Leveduras } \\
\text { 3,92690a }\end{array}$ & $3,38640 \mathrm{a}$ & $25,08 \%$ \\
\hline
\end{tabular}

*Médias seguidas de letras minúsculas distintas nas colunas diferem entre si pelo teste de Tukey $(\mathrm{p} \leq 0,05)$.

\section{CONCLUSÃO}

Leveduras e bolores estão claramente associados ao processo de decomposição de matéria orgânica vegetal ao longo de todo o período de estudo, indicando um papel importante neste processo em córregos.

$\mathrm{O}$ papel preponderante dos fungos $\mathrm{e}$ leveduras é exercido durante o processo inicial de 30 dias de decomposição de matéria orgânica vegetal.

O período chuvoso promove o aumento das contagens de fungos filamentosos e leveduras associados ao detrito foliar.

As populações de bolores apresentam comportamento sazonal, já que as contagens de bolores apresentaram diferença estatisticamente significativa a $1 \%$. O mesmo não ocorre com as contagens de leveduras, que não apresentam diferença estatisticamente significativa, comparando-se os períodos de seca e de chuva.

\section{AGRADECIMENTO}

Ao $\mathrm{CNPq}$ e Capes pelo financiamento deste trabalho.

\section{CONTRIBUIÇÃO DOS AUTORES}

1. Condução e avaliação do experimento, análises estatísticas e elaboração do artigo:

$\mathrm{Dr}^{\mathrm{a}}$. Anelise Kappes Marques

Jessica Barros Aguiar Silva

Amaraina Armiato Maia

Laciene Marinho dos Santos

2. Planejamento, orientação e revisão final do artigo:

Dra . Paula Benevides de Morais

\section{REFERÊNCIAS}

[1]. ALMEIDA, D. A. C.; BARBOSA, F. R.; GUSMÃO, L. F. P. Alguns fungos conidiais aquáticos-facultativos do bioma Caatinga. Acta Botanica Brasilica v. 26, n.4, p. 924-932, 2012. DOI:10.1590/S0102-33062012000400021

[2]. GRAÇA, M.A.S.; ZIMMER, M. Leaf toughness. In: GRAÇA, M.A.S.; BÄRLOCHER F.; GESSNER, M.O. (Eds.). Methods to study litter decomposition: a practical guide, Springer. 2005. p.121-125

[3]. ALEMANNO, S.; MANCINELLI, G.; BASSET, A. Effects of invertebrate patch use behaviour and detritus quality on reed leaf decomposition in aquatic systems: A modelling approach. Ecological Modelling, v.205, p.492-506, 2007. DOI:10.1016/j.ecolmodel.2007.03.009

[4]. GONÇALVES JR., J.F. Decomposição de detritos foliares em riachos: composição química, invertebrados e microrganismos. 2005. Tese (Doutorado) - Universidade Federal de Minas Gerais, Belo Horizonte

[5]. SHEARER, C. A.; DESCALS, E.; KOHLMEYER, B.; KOHLMEYER J.;
MARVANOVA, L.; PADGETT, D.; PORTER, D.; RAJA, H. A.; SCHMIT, J. P.; THORTON, H. A.; VOGLYMAYR, H. Fungal biodiversity in aquatic habitats Biodiversity and Conservation, v. 16 p. $49-67,2007$. DOI:10.1007/s/0531-0069120-z

[6]. AleXopoulos, C. J.; MIMS, C. W.; BLACKWELL, M. Introductory Mycology. New York: John Wiley \& Sons, 1996. 869p.

[7]. BONATTO, G. Uso da terra e produção de sedimentos na sub-bacia do ribeirão Taquaruçu Grande, contribuinte ao reservatório da usina hidrelétrica do Lajeado, Tocantins. 2008. 96p. Dissertação (Mestrado em Geociências). Universidade Federal do Rio de Janeiro, Rio de Janeiro.

[8]. MARQUES, A. K.; COTRIM, M. A. B.; BARROS, D. J.; BONATTO, G.; PIRES, M. A. F. Avaliação da qualidade da água do ribeirão Taquaruçu Grande e do reservatório da UHE Lajeado, médio Tocantins, Brasil, Sodebras, vol.10, n.117, p. 23-29, 2015.

[9]. GONÇALVES JR., J. F.; CALliSTO, M. Organic-matter dynamics in the riparian zone of a tropical headwater stream in Southern Brasil. 
Aquatic Botanic, v.109, p.8-13, 2013 DOI:10.1016/j.aquabot.2013.03.005

[10]. GONÇALVES Jr, J. F.; FRANÇA, J. S.; MEDEIROS, A. O.; ROSA, C. O.; CALLISTO, M. Leaf breakdown in a tropical stream. International Review Hydrobiology, v.91, p.164-177, 2006. DOI:10.1002/iroh.200510826

[11]. NEDER, R. N. Microbiologia - Manual de Laboratório. São Paulo:Nobel. 1992. 138p

[12]. SILVA, F. A. S.; AZEVEDO, C. A. V. Principal components Analysis in the software AssistatStatistical Attendence. In: WORLD CONGRESS ON COMPUTER IN AGRICULTURE 7, RenoNV-USA: Anais... American Society of Agricultural and Biological Engeneers. 2009.

[13]. SILVA-BEDOYA L.M., RAMÍREZCASTRILLÓN M., OSORIO-CADAVID E. Yeast diversity associated to sediments and water from two Colombian artificial lakes. Brazilian
Journal of Microbiology, v.45, n.1, p.135-142, 2014. DOI:10.1590/S1517-83822014005000035

[14]. WANTZEN, K. M.; YULE, C. M.; MATHOOKO, J. M.; PRINGLE, C. M. Organic matter processing in tropical streams. Tropical Stream Ecology, v.1, p.44-65, 2008

[15]. SCHOENLEIN-CRUSIUS, I. H.; MOREIRA, C. G.; TAKAHASHI, J. P. GOMES, E. P. C. Riqueza dos fungos ingoldianos e aquáticos facultativos no Parque Municipal do Ibirapuera, São Paulo, SP, Brasil. Hoehnea, v.41, p.61-76, 2014.

[16]. GRAÇA, M. A. S.; CANHOTO, C. Leaf litter processing in low order streams. Limnetica, v.25, p.1-10, 2006.

[17]. SCHOENLEIN-CRUSIUS, I. H.; MOREIRA, C. G., BICUDO, D. C. Aquatic Hyphomycetes in the Parque Estadual das Fontes do Ipiranga. Revista Brasileira de Botânica v.32, n.3, p.411-426, 2009. 